\title{
Turbulence Fluctuations and New Universal Realizability Conditions in Modelling
}

\author{
Gregory L. Eyink \\ Department of Mathematics \\ University of Arizona \\ Tucson, AZ 85721 \\ and \\ Francis J. Alexander \\ Center for Computational Science \\ 3 Cummington Street \\ Boston University \\ Boston, MA 02215 \\ (November 21, 2018)
}

General turbulent mean statistics are shown to be characterized by a variational principle. The variational functionals, or "effective actions", have experimental consequences for turbulence fluctuations and are subject to realizability conditions of positivity and convexity. An efficient Rayleigh-Ritz algorithm is available to calculate approximate effective actions within PDF closures. Examples are given for Navier-Stokes and for a 3-mode system of Lorenz. The new realizability conditions succeed at detecting a priori the poor predictions of PDF closures even when the classical 2nd-order moment realizability conditions are satisfied.

PACS Numbers: 47.27.-i, 47.27.Sd, 47.11.+j

It does not seem to be a well-recognized fact that general turbulence mean statistics - such as mean velocity or pressure profiles - are characterized by a variational principle. However, in nonequilibrium statistical mechanics it was pointed out long ago by Onsager [1.22 that mean histories satisfy a "principle of least action". The so-called Onsager-Machlup action determines the probability of fluctuations away from the most probable state. Close to thermal equilibrium there is a standard fluctuationdissipation relation, so that the action has the physical interpretation of a "dissipation function". Onsager's variational principle reduces then to a principle of least dissipation.

Recently it has been pointed out by one of us 3. 3 . that a similar effective action $\Gamma[z]$ exists in turbulent flow for any random variable $z(t)$. This action function (i) is non-negative, $\Gamma[z] \geq 0$, (ii) has the ensemble mean $\bar{z}(t)$ as its unique minimum $\Gamma[\bar{z}]=0$, and (iii) is convex, $\Gamma\left[\lambda z_{1}+(1-\lambda) z_{2}\right] \geq \lambda \Gamma\left[z_{1}\right]+(1-\lambda) \Gamma\left[z_{2}\right], 0<$ $\lambda<1$. These are realizability conditions which arise from positivity of the underlying statistical distributions. As a consequence, the mean value $\bar{z}(t)$ is characterized by a "principle of least effective action". Just as Onsager's action, this functional is related to fluctuations. In particular, in statistically stationary turbu- lence, the time-extensive limit of the effective action, $V[z] \equiv \lim _{T \rightarrow \infty} \frac{1}{T} \Gamma[\{z(t)=z: 0<t<T\}]$, the so-called effective potential, determines the probability of fluctuations in the empirical time-average $\bar{z}_{T} \equiv \frac{1}{T} \int_{0}^{T} d t z(t)$ away from the (time-independent) ensemble-mean value $\bar{z}$. More precisely, the probability for any value $z$ of the time-average $\bar{z}_{T}$ to occur is given by

$$
\operatorname{Prob}\left(\left\{\bar{z}_{T} \approx z\right\}\right) \sim \exp (-T \cdot V[z]) .
$$

This agrees with the standard ergodic hypothesis, according to which, as $T \rightarrow \infty$, the empirical time-average must converge to the ensemble-mean, $\bar{z}_{T} \rightarrow \bar{z}$, with probability one in every flow realization. The Eq.(11) refines that hypothesis, by giving an exponentially small estimate of the probability at a large (but finite) $T$ to observe fluctuations away from the ensemble-mean.

The realizability conditions on the effective action or effective potential hold even when there are no classical 2nd-moment realizability conditions on the means themselves. Thus, energy spectra or Reynolds stresses (2nd moments) must be positive, but mean velocity profiles (1st moments) or energy transfer (3rd moments) do not satisfy such simple realizability conditions. The new realizability conditions thus have great potential significance in modelling if they can be efficiently calculated within turbulence closures. In [3, [n] we have shown that there is a simple Rayleigh-Ritz algorithm within PDF closuressuch as mapping closures [5, 6] or generalized Langevin models [7,8] - by which the corresponding approximate values of the effective action or effective potential may be readily calculated.

As a simple example, we consider first a 3 -mode system of Lorenz [9], in which the equations of motion are

$$
\dot{x}_{i}=A_{i} x_{j} x_{k}-\nu_{i} x_{i}+f_{i}
$$

with $i, j, k$ a cyclic permutation of $1,2,3$, with $A_{1}+$ $A_{2}+A_{3}=0$ imposed on interaction coefficients $A_{i}$ for energy conservation, with $\nu_{i}$ positive damping coefficients, and $f_{i}(t)$ white-noise random forces with covariance $2 \kappa_{i}$. This dyamics has been used often as a first test 
of closure ideas [10]- 12]. We consider a simple mapping closure proposed by Bayly for the 3-mode system [13], which models the realizations by a quadratic map $X_{i}=\beta_{i} N_{i}+\beta_{4} N_{j}^{\prime} N_{k}^{\prime}$ of independent standard Gaussian variables $N_{i}, N_{i}^{\prime}, i=1,2,3$. The resulting closure equations for the 2 nd moments $M_{i}=\left\langle x_{i}^{2}\right\rangle, i=1,2,3$ and the 3rd moment $T=\left\langle x_{1} x_{2} x_{3}\right\rangle$ are

$$
\dot{M}_{i}=2 A_{i} T-2 \nu_{i} M_{i}+2 \kappa_{i}
$$

for $i=1,2,3$ and

$$
\begin{gathered}
\dot{T}=A_{1} M_{2} M_{3}+A_{2} M_{1} M_{3}+A_{3} M_{1} M_{2} \\
-\left(\nu_{1}+\nu_{2}+\nu_{3}\right) T .
\end{gathered}
$$

These are just the quasinormal (QN) equations for the 3-mode system, obtained by neglecting the 4th-order cumulants [14]. It was already noted by Kraichnan 10] that, unlike for Navier-Stokes, the QN closure for the 3mode system predicts all positive energies. In fact, for $A_{1}=2, A_{2}=A_{3}=-1, \kappa_{1}=1, \kappa_{2}=\kappa_{3}=0.001, \nu_{1}=$ $0.001, \nu_{2}=\nu_{3}=1$ it gives steady-state values

$$
\begin{gathered}
M_{1}^{(Q N)} \approx 1.49875, M_{2}^{(Q N)}=M_{3}^{(Q N)} \approx 0.50025 \\
T^{(Q N)} \approx-0.49925 .
\end{gathered}
$$

All of the 2nd moments are positive, as required by realizability. However, DNS of the 3-mode dynamics itself gives

$$
\begin{aligned}
& M_{1}^{(D N S)}=4.46 \pm 0.03 \\
& M_{2}^{(D N S)}=M_{3}^{(Q N)}=0.49876 \pm 0.00002 \\
& T^{(D N S)}=-0.49776 \pm 0.00002
\end{aligned}
$$

While the QN predictions for $M_{2}, M_{3}$ and $T$ are within $\frac{1}{3} \%$ of the DNS values, $M_{1}$ is underpredicted by $66 \%$ in the QN approximation. As is well-known, satisfaction of realizability cannot guarantee that a prediction is correct. However, failure of realizability certainly implies that the predictions are in error. In Figs.1-3 we graph the approximate effective potentials of the energy variables $E_{i}=\frac{1}{2} x_{i}^{2}$ and triple product $T=x_{1} x_{2} x_{3}$ in the $Q N$ closure as calculated by the Rayleigh-Ritz algorithm for the above PDF model. The numerical method is outlined below and described in detail in [4,15. It is apparent that $V_{E_{2}}$ and $V_{T}$ satisfy realizability but that $V_{E_{1}}$ does not. Thus, one may infer a priori - without knowledge of the DNS results - that the QN prediction for the mean of $E_{1}$ is not converged. In this case, the failure of realizability of the predicted $V_{E_{1}}$ succeeds at detecting the poor prediction for the mean value, even though the classical 2nd-moment condition $E_{1} \geq 0$ is satisfied. In the same plots in Figs.2-3 we have graphed also the effective potentials $V_{E_{2}}$ and $V_{T}$ obtained from DNS. They do not agree with the QN potentials as closely as do the corresponding means: the accurate prediction of fluctuations is a much more stringent demand on the closure. However, we note that the predictions of Bayly's PDF closure are at least qualitatively correct for $V_{E_{2}}$ and $V_{T}$ and give correctly the order of magnitude of the averaging-time needed to eliminate fluctuations in those variables. Of course, the prediction of $V_{E_{1}}$ is not even qualitatively correct.

The Rayleigh-Ritz algorithm used in obtaining the approximate potentials from the PDF closure involves a fixed point problem very similar to (and, in fact, generalizing) the fixed point condition determining the predicted steady-state moments themselves. The system of equations that must be solved in general is

$$
\begin{gathered}
\frac{\partial V_{0}}{\partial \mathbf{M}}(\mathbf{M}, \mathbf{h}) \alpha_{0}+\left(\frac{\partial \mathbf{V}}{\partial \mathbf{M}}\right)^{\top}(\mathbf{M}, \mathbf{h}) \cdot \boldsymbol{\alpha}=V_{0}(\mathbf{M}, \mathbf{h}) \boldsymbol{\alpha} \\
\mathbf{V}(\mathbf{M}, \mathbf{h})=V_{0}(\mathbf{M}, \mathbf{h}) \mathbf{M} \\
\alpha_{0}+\boldsymbol{\alpha} \cdot \mathbf{M}=1
\end{gathered}
$$

The vector $\mathbf{M}=\left(M_{1}, \ldots, M_{k}\right)$ contains the moments of the closure, e.g., in the case above, $k=4\left(\right.$ and $\left.M_{4}=T\right)$. $\mathbf{h}$ is the vector of "perturbation fields", one associated with each variable $Z_{i}$ for which the potential is to be determined. In our previous calculation $\mathbf{h}=\left(h_{E_{1}}, h_{E_{2}}, h_{T}\right)$. When $\mathbf{h}=\mathbf{0}$ the vector $\mathbf{V}(\mathbf{M}, \mathbf{h})$ coincides with the dynamical vector $\mathbf{V}(\mathbf{M})$ which appears in the closure equation: $\dot{\mathbf{M}}=\mathbf{V}(\mathbf{M})$ (cf. Eqs.(3),(1) above). The perturbations for $\mathbf{h} \neq \mathbf{0}$ are determined by the method discussed in [4]. The 0-component $V_{0}(\mathbf{M}, \mathbf{h})$ is associated with the zeroeth moment $M_{0} \equiv 1$ and it may be written explicitly here as $V_{0}(\mathbf{M}, \mathbf{h})=\frac{1}{2} h_{E_{1}} M_{1}+\frac{1}{2} h_{E_{2}} M_{2}+h_{T} M_{4}$. It is easy to check that, when $\mathbf{h}=\mathbf{0}$, the stationary moments $\mathbf{M}_{*}$ along with $\alpha_{* 0}=1, \boldsymbol{\alpha}_{*}=\mathbf{0}$ solve the system Eqs.(7)(9). Once the solutions $\alpha_{* 0}(\mathbf{h}), \boldsymbol{\alpha}_{*}(\mathbf{h}), \mathbf{M}_{*}(\mathbf{h})$ are known for $\mathbf{h} \neq \mathbf{0}$, the effective potential $V_{Z_{i}}$ is constructed as a function of $h_{i}$ via $V_{Z_{i}}\left[h_{i}\right]=-\boldsymbol{\alpha}_{*}\left(h_{i}\right) \cdot \mathbf{V}\left(\mathbf{M}_{*}\left(h_{i}\right)\right)$. To obtain the potential as a function of $z_{i}$, the expected value $\mathbf{Z}_{*}(\mathbf{h})=\mathbf{z}$ must be inverted to give $h_{i}$ as a function of $z_{i}$. For full details of the algorithm, see 14 .

Our results point toward significant new directions in turbulence modelling. The new realizability conditions apply individually to all predicted means. We see above that they can successfully discriminate between poor predictions for one set of variables and good predictions for another. Calculating each point on the graph of an effective potential curve within a closure requires just the same amount of computation as that to calculate the predicted mean. It is therefore very easy to apply the above realizability conditions as a check to detect poor predictions in advance, without expensive testing by experiment or simulation. This gives a strong incentive to the development of PDF closures, such as those in Refs. [5]- [8]. In conjunction with our variational method they can give some a priori information in turbulence modelling. This is a unique advantage, almost never obtained in other statistical closure methods. 
It remains to be seen how well the new realizability conditions succeed in detecting poor predictions of closures for Navier-Stokes turbulence. It is thus worthwhile to give one example demonstrating the Rayleigh-Ritz method for a statistically time-dependent Navier-Stokes flow. The simplest such situation is freely-decaying homogeneous and isotropic turbulence with random initial data. We consider a model energy spectrum

$$
E(k, t)= \begin{cases}A k^{m} & k \leq k_{L}(t) \\ \alpha \varepsilon^{2 / 3}(t) k^{-5 / 3} & k_{L}(t) \leq k \leq k_{d}(t) \\ 0 & k \geq k_{d}(t)\end{cases}
$$

which has been adopted before in this problem [16, 17. As long as $0<m<4$ it is commonly believed that there is a permanence of the low-wavenumber spectrum. This motivates one to adopt the above self-preserving form, in which the shape of the spectrum is unchanged in time except through its dependence on the parameters $\varepsilon(t), k_{L}(t)$ and $k_{d}(t)$. At high Reynolds number there is only one independent such parameter, since the relation $k_{L}(t)=\left(\frac{\alpha}{A} \varepsilon(t)\right)^{\frac{3}{3 m+5}}$ is required by continuity and, when $k_{L}(t) \ll k_{d}(t), k_{d}(t)=\left(\frac{4}{3 \alpha \nu}\right)^{3 / 4} \varepsilon^{1 / 4}(t)$ also holds [17]. The remaining time-dependence is determined by considering the evolution of the mean energy $E(t)=\frac{1}{2}\left\langle v^{2}(t)\right\rangle$. For the above form of the spectrum it is not hard to show [17] that the dissipation $\varepsilon(t)=\frac{\nu}{2} \sum_{i j}\left\langle\left(\partial_{i} v_{j}+\partial_{j} v_{i}\right)^{2}\right\rangle$ is given as

$$
\varepsilon(t)=\Lambda_{m} \cdot E^{p}(t)
$$

with $\Lambda_{m}^{-1}=\alpha^{3 / 2}\left(\frac{1}{m+1}+\frac{3}{2}\right)^{\frac{3 m+5}{2 m+2}} A^{\frac{1}{m+1}}$ and $p=\frac{3 m+5}{2 m+2}$. Thus, employing the Navier-Stokes equation via its energy-balance, one obtains the closed equation

$$
\dot{E}(t)=-\Lambda_{m} \cdot E^{p}(t) .
$$

Its solution gives a prediction for the energy-decay law, as $E_{*}(t) \sim\left(t-t_{0}\right)^{-\frac{2 m+2}{m+3}}$ : see [17].

It is interesting to make a check on the various hypotheses involved in these predictions by means of the effective action $\Gamma[E]$ for the energy history $E(t)$. As a simple PDF model for the above closure, one may adopt a Gaussian random velocity field with the assumed selfsimilar spectrum Eq.(10). The Rayleigh-Ritz approximation of the effective action within the Gaussian ansatz can be analytically evaluated [18], with the result:

$$
\begin{aligned}
& \Gamma^{(\text {Gauss })}[E]=\frac{3}{2(p-2) \Lambda_{m}} \times \\
& \int_{0}^{\infty} d t \frac{\left(\dot{E}(t)+\Lambda_{m} \cdot K^{p}(t)\right)\left(\dot{K}(t)+\Lambda_{m} \cdot K^{p}(t)\right)}{K^{p+1}(t)}
\end{aligned}
$$

where $K(t)$ is a variational parameter satisfying

$$
\Lambda_{m} \cdot K^{p}(t)+\dot{E}(t)=
$$

$$
(p-2) \Lambda_{m} \cdot(E(t)-K(t)) \cdot K^{p-1}(t) .
$$

It is easy to check that, if the predicted closure mean energy $E_{*}(t)$ satisfying $\dot{E}_{*}(t)=-\Lambda_{m} E_{*}^{p}(t)$ is substituted, then $\Gamma^{(\text {Gauss })}\left[E_{*}\right]=0$. Further insight is obtained by considering small perturbations $E(t)=E_{*}(t)+\delta E(t)$ from the predicted mean. By a straightforward calculation it follows that

$$
\begin{aligned}
& \Gamma^{(\text {Gauss })}[E]=\frac{3}{8(p-1) \Lambda_{m}} \times \\
& \int_{0}^{\infty} d t \frac{\left(\delta \dot{E}(t)+\Lambda_{m} \cdot p E_{*}^{p-1}(t) \delta E(t)\right)^{2}}{E_{*}^{p+1}(t)}+O\left(\delta E^{3}\right) .
\end{aligned}
$$

This is the same law of fluctuations as would be realized with the Langevin equation

$$
\delta \dot{E}(t)+\Lambda_{m} \cdot p E_{*}^{p-1}(t) \delta E(t)=\sqrt{2 R_{*}(t)} \eta(t)
$$

obtained by linearization of the energy-decay equation around its solution $E_{*}(t)$ and by addition of a white-noise random force $\eta(t)$ with strength

$$
R_{*}(t)=\frac{2(p-1)}{3} \varepsilon_{*}(t) E_{*}(t) .
$$

Thus, the smaller fluctuations from the ensemble-mean value are predicted to decay according to a linearized law, similar to the Onsager regression hypothesis for equilibrium fluctuations. Likewise, the expression Eq.(17) is a fluctuation-dissipation relation analogous to that in equilibrium. These are testable predictions of the Gaussian closure. Note that the coefficient $(p-1)$ in front of the action is $>0$ as long as $m>-3$. In fact, $m>-1$ is required to give a finite energy. Thus, for all permissable values of $m$, the approximate action $\Gamma^{(\text {Gauss })}[E]$ satisfies realizability. One should be cautioned again that satisfaction of realizability is only a consistency check and cannot guarantee correctness of predictions. Failure of realizability, as observed in the 3-mode model, is more practically useful, although in a purely negative way.

The previous examples and our variational method are discussed in greater detail in forthcoming papers [15, 18]. Here, we have simply wished to illustrate briefly the use of the action principle. Future work will study the success of the new realizability conditions in detecting poor closure predictions for more realistic Navier-Stokes flows, of greater interest to practical engineering. It should be clear that very general PDF ansatz may be employed in our method, either by guessing a functional form of the PDF or by hypothesizing "surrogate" random variables to model the actual flow realizations. Any guess of the turbulence statistics - such as the "synthetic turbulence" models of 19] - may be input to yield predictions for realistic problems. We therefore expect our method to be a flexible framework within which to develop novel turbulence closures. Insights from simulation, experiment 
and recent theoretical developments can be readily incorporated. The advantage of the variational formulation is that it provides built-in checks of statistical closures which may detect a sizable fraction of faulty predictions in advance. By doing so cheaply, it can provide great savings in turbulence modelling for practical engineering purposes.

Acknowledgements: We wish to thank R. H. Kraichnan for his interest in and encouragement of this work. Numerical computations were carried out at the Center for Computational Science at Boston University and the Department of Mathematics at the University of Arizona.

\section{FIGURE CAPTIONS}

Figure 1.) Effective potential for energy in mode 1 in quasinormal closure.

Figure 2.) Effective potential for energy in mode 2 in quasinormal closure. (DNS with errorbars).

Figure 3.) Effective potential for triple moment in quasinormal closure. (DNS with errorbars).
[1] L. Onsager, Phys. Rev. 37405 (1931); 382265 (1931).

[2] L. Onsager and S. Machlup, Phys. Rev. 911505 (1953).

[3] G. L. Eyink, J. Stat. Phys. 83955 (1996).

[4] G. L. Eyink, Phys. Rev. E, to appear (1996), chaodyn/9505001.

[5] H. Chen, S. Chen and R. H. Kraichnan, Phys. Rev. Lett. 65575 (1990).

[6] T. Gotoh and R. H. Kraichnan, Phys. Fluids A 5445 (1992).

[7] D. C. Haworth and S. B. Pope, Phys. Fluids 29387 (1986).

[8] S. B. Pope, Ann. Rev. Fluid Mech. 2623 (1994).

[9] E. Lorenz, Tellus 12243 (1960).

[10] R. H. Kraichnan, Phys. Fluids 61603 (1963).

[11] R. H. Kraichnan, in: Dynamics of Fluids and Plasmas, ed. S. I. Pai (Academic Press, New York, 1966), pp.239255.

[12] S. A. Orszag and L. R. Bissonnette, Phys. Fluids 102603 (1967).

[13] B. Bayly, unpublished (1992).

[14] I. Proudman and W. H. Reid, Phil. Trans. Roy. Soc. A 247163 (1954).

[15] G. L. Eyink and F. J. Alexander, in preparation.

[16] G. Comte-Bellot and S. Corrsin, J. Fluid Mech. 25657 (1966).

[17] W. C. Reynolds, Ann. Rev. Fluid Mech. 8183 (1976).

[18] G. L. Eyink, in preparation (1996).

[19] A. Juneja et al. Phys. Rev. E 495179 (1994). 


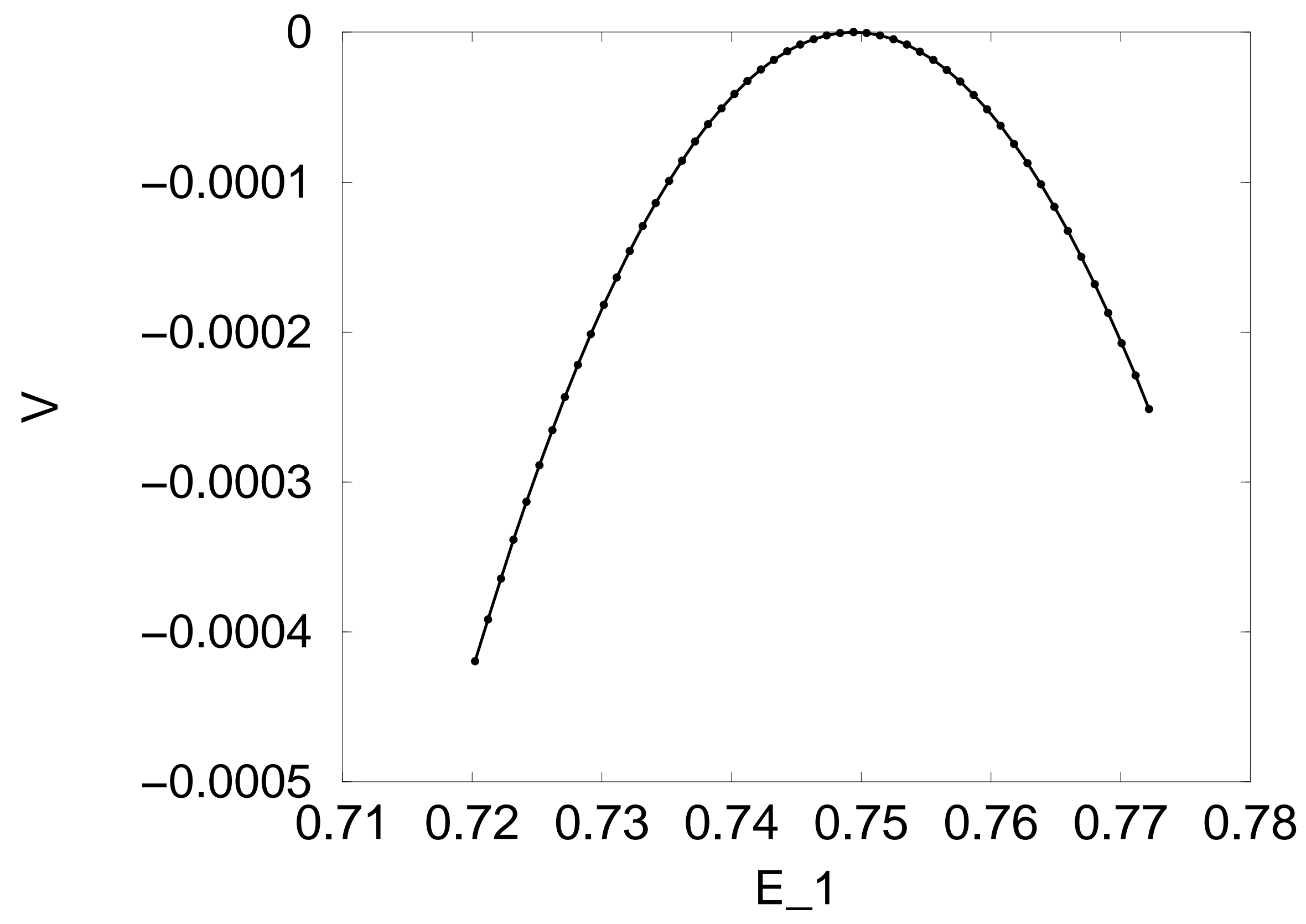




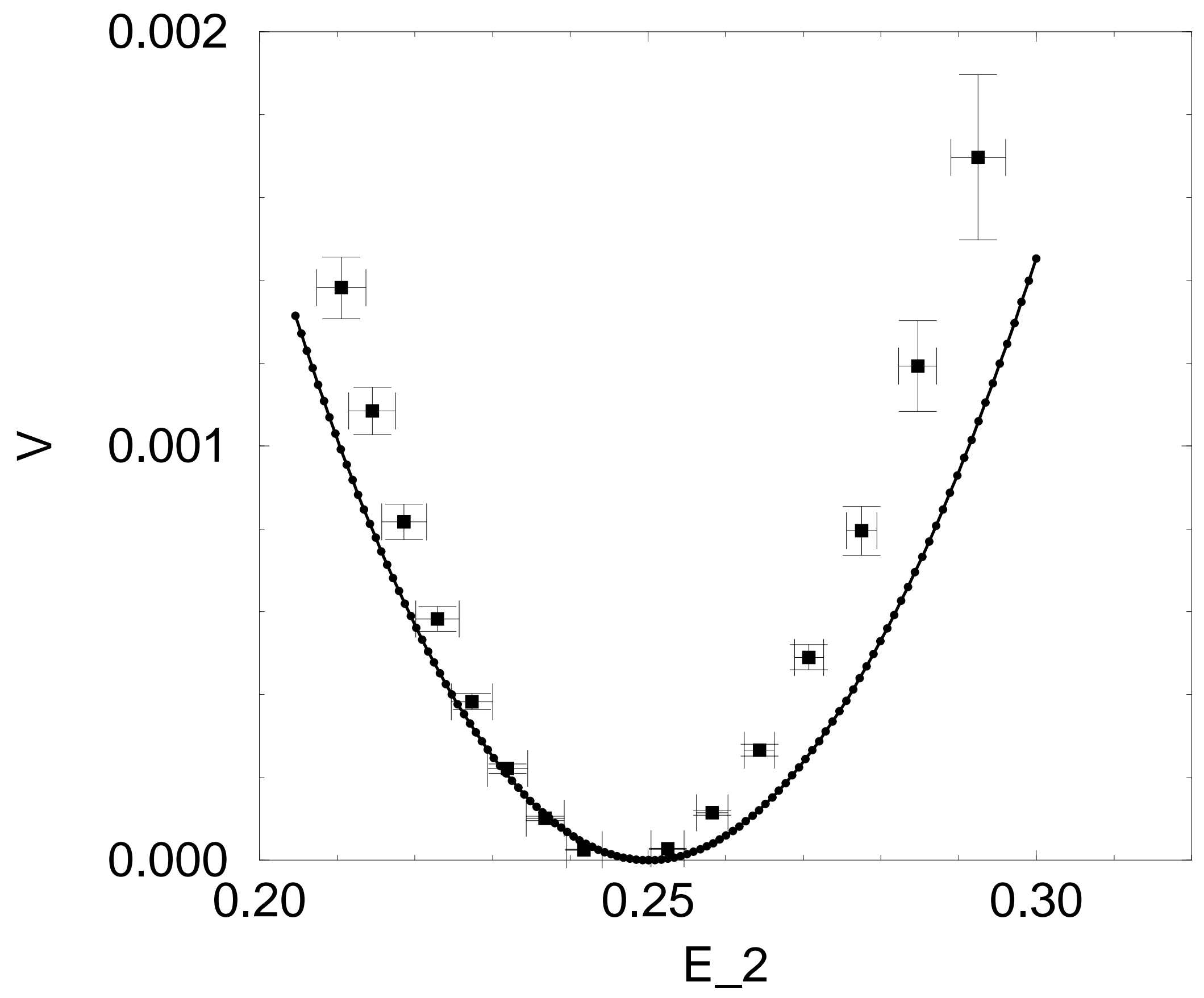




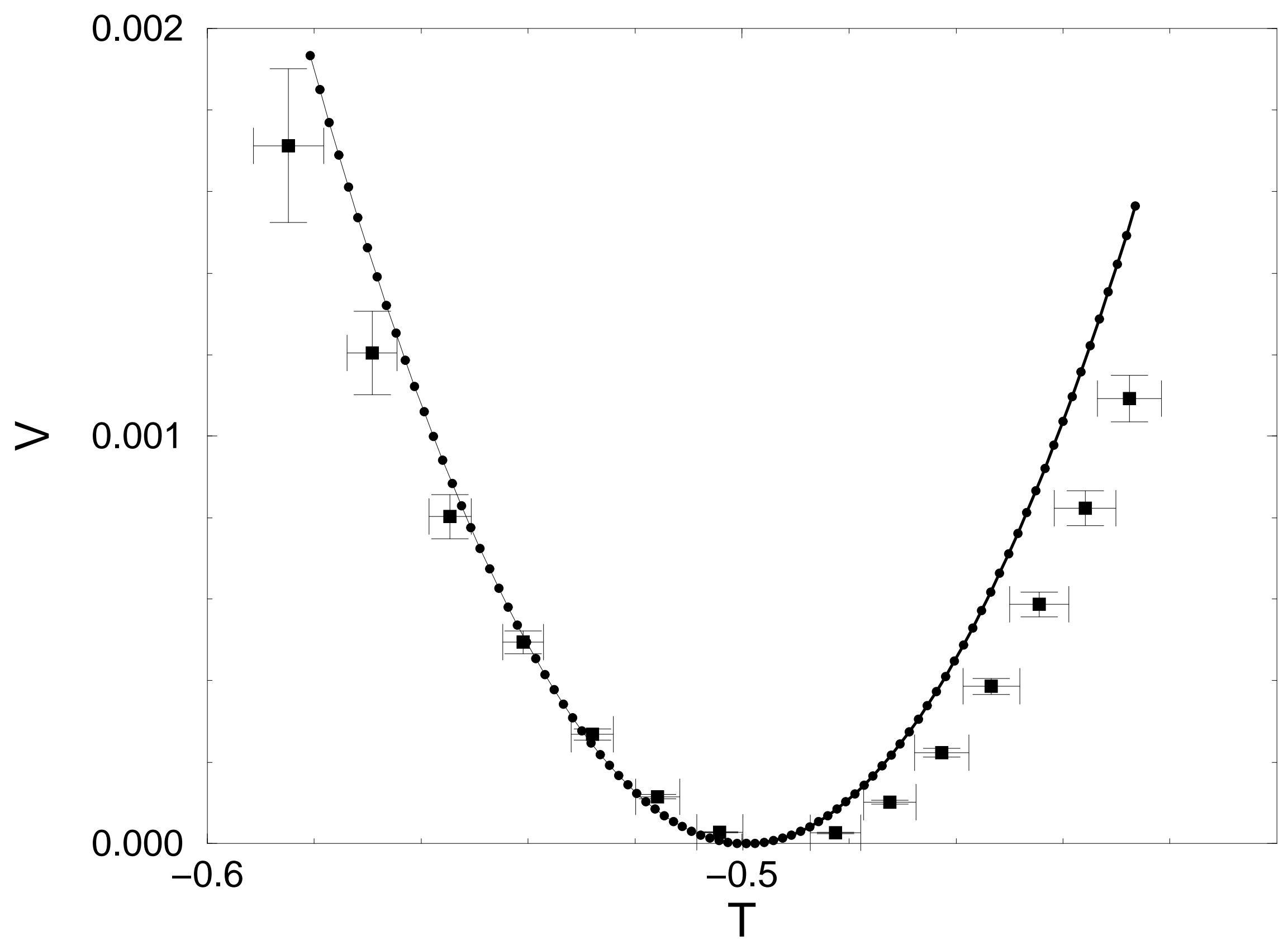

